\title{
Playing games with epigenetics
}

The CDKN2A locus encodes the tumour suppressors INK4A and ARF, which induce senescence through independent pathways. $C D K N 2 A$ expression is repressed by histone $\mathrm{H} 3$ lysine 27 trimethylation (H3K27me3), which is mediated by polycomb group proteins. This repression is reversed in response to cellular stress, such as oncogene activation, to induce senescence. Two papers in Genes \& Development now provide evidence that the histone lysine demethylase JMJD3 (also known as KDM6B) removes H3K27me3 from CDKN2A and may therefore have an important role in mediating senescence as a barrier to tumorigenesis.

Karl Agger and colleagues assessed the gene expression changes of transformed diploid fibroblasts undergoing oncogeneinduced senescence (mediated by HRAS-V12 or BRAF expression) to identify factors that could be responsible for the de-repression of CDKN2A.

Real-time quantitative PCR revealed that JMJD3 expression increased with oncogene expression before INK4A was expressed, consistent with transcriptional activation of
CDKN2A following demethylation. Supporting this, knockdown of JMJD3 reduced INK4A expression following BRAF expression. In addition, expression of a catalytically active fragment of JMJD3 in mouse embryonic fibroblasts induced INK4A without oncogene expression. These cells exhibited reduced proliferation, indicating that JMJD3 is sufficient to induce INK4Adependent senescence, and further examination revealed that this correlates with the loss of $\mathrm{H} 3 \mathrm{~K} 27 \mathrm{me} 3$ on the CDKN2A INK4A locus. The authors also found that ultraviolet irradiation increased JMJD3 expression, indicating that other types of stress might induce senescence through JMJD3.

In the second paper, Barradas, Anderton, Acosta and colleagues assessed the $\mathrm{H} 3 \mathrm{~K} 27 \mathrm{me} 3$ status of CDKN2A using chromatin immunoprecipitation and found that H3K27me3 decreased with the induction of HRAS-V12-mediated senescence. This correlated with increased JMJD3 expression and binding to the $C D K N 2 A$ locus, accompanied by loss of EZH2, the polycomb group lysine methyltransferase responsible for $\mathrm{H} 3 \mathrm{~K} 27$ methylation. Knockdown of JMJD3 partially restored cell cycling and reduced INK4A expression, whereas ectopic expression of full-length or catalytically active fragments of JMJD3 induced INK4A expression and features of

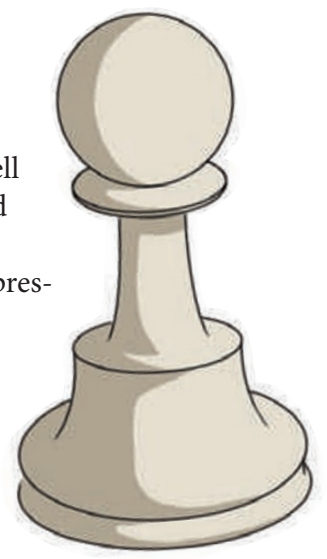
senescence.

Therefore, JMJD3 directly binds to and de-represses the CDKN2A locus, allowing INK4A expression and mediating oncogene-induced senescence. Searches in the Oncomine database by both groups revealed that JMJD3 expression is significantly reduced in several lymphomas and upregulated in early and benign lesions but absent in more advanced tumours, suggesting that JMJD3-mediated senescence serves as an early barrier to tumorigenesis.

Gemma K. Alderton

ORIGINAL RESEARCH PAPERS Agger, K. et al. The H3K27 me3 demethylase JMJD3 contributes to the activation of the INK4A-ARF locus in response to oncogene- and stress-induced senescence. Genes Dev. 23, 1171-1176 (2009)| Barradas, M. et al. Histone demethylase JMJD3 contributes to epigenetic control of INK4a/ARF by oncogenic RAS. Genes Dev. 23, 1177-1182 (2009) 